International Journal of Pure and Applied Mathematics

Volume 103 No. 2 2015, 235-241

ISSN: 1311-8080 (printed version); ISSN: 1314-3395 (on-line version)

url: http://www.ijpam.eu

doi: http://dx.doi.org/10.12732/ijpam.v103i2.9

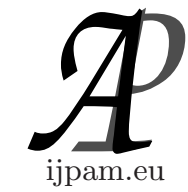

\title{
A QUINTINC POLYNOMIAL DIFFERENTIAL SYSTEMS WITH EXPLICIT NON-ALGEBRAIC LIMIT CYCLE
}

\author{
Rachid Boukoucha ${ }^{1}$, Ahmed Bendjeddou ${ }^{2}$ \\ ${ }^{1}$ Department of Mathematics \\ Faculty of Sciences Exactes and Informatics \\ University of Jijel \\ 18000 Jijel, ALGERIA \\ ${ }^{2}$ Departement de Mathematics \\ Faculty of Sciences \\ University of Setif \\ 19000 Setif, ALGERIA
}

\begin{abstract}
In this paper, we introduce a multi-parameter quintinc polynomial differential system for which we prove the existence of a non-algebraic limit cycle. Moreover this limit cycle is explicitely given in polar coordinates. Concrete examples exhibiting the applicability of our result are introduced.
\end{abstract}

AMS Subject Classification: 34C29, 34C25

Key Words: planar polynomial differential system, non-algebraic limit cycle

\section{Introduction}

An important problem of the qualitative theory of differential equations is to determine the limit cycles of a system of the form

$$
\left\{\begin{array}{l}
x^{\prime}=P(x, y) \\
y^{\prime}=Q(x, y)
\end{array}\right.
$$

where $P(x, y)$ and $Q(x, y)$ are coprime polynomials and we denote by $n=$ $\max \{\operatorname{deg} P, \operatorname{deg} Q\}$ and we say that $n$ is the degree of system 1.1, see [5]. A

Received: April 9, 2015

(C) 2015 Academic Publications, Ltd.

$\S$ Correspondence author url: www.acadpubl.eu 
limit cycle of system 1.1 is an isolated periodic orbit and it is said to be algebraic if it is contained in the zero level set of a polynomial function, see for example, $[2,3,8]$, and [9] . We usually only ask for the number of such limit cycles, but their location as orbits of the system is also an interesting problem. And an even more difficult problem is to give an explicit expression of them. We are able to solve this last problem for a given system of the form 1.1. Until recently, the only limit cycles known in an explicit way were algebraic. In $[1$, $6]$, and [7] examples of explicit limit cycles which are not algebraic are given. For instance, the limit cycle appearing in van der Pol's system is not algebraic as it is proved in [10].

In this paper, we introduce a multi-parameter quintinc polynomial differential system of the form

$$
\left\{\begin{aligned}
x^{\prime} & =x\left((m+l) x^{2}+2 n x y+(m-l) y^{2}\right) \\
& +(x-2 y)\left(x^{2}+y^{2}\right)\left((a+c) x^{2}+2 b x y+(a-c) y^{2}\right) \\
y^{\prime} & =y\left((m+l) x^{2}+2 n x y+(m-l) y^{2}\right) \\
& +(2 x+y)\left(x^{2}+y^{2}\right)\left((a+c) x^{2}+2 b x y+(a-c) y^{2}\right)
\end{aligned}\right.
$$

where $a, b, c, n, m, l$ real constants.

We prove the existence of a non-algebraic limit cycle. Moreover this limit cycle is explicitly given in polar coordinates. Concrete examples exhibiting the applicability of our result are introduced.

\section{The Main Result}

Our main result is contained in the following theorem

Theorem 1. The multi-parameter polynomial differential system 1.2, has non-algebraic limit cycle explicitly given in polar coordinates $(r, \theta)$ by :

$$
r(\theta)=e^{\frac{1}{2} \theta} \sqrt{r_{*}^{2}+\int_{0}^{\theta}\left(e^{-s} \frac{m+l \cos 2 s+n \sin 2 s}{a+c \cos 2 s+b \sin 2 s}\right) d s}
$$

such that $a+|c|+|b|<0, m-|l|-|n|>0$, and

$$
r_{*}=\sqrt{\frac{e^{2 \pi}}{-1+e^{2 \pi}}} \sqrt{\int_{0}^{2 \pi}-\left(e^{-s} \frac{m+l \cos 2 s+n \sin 2 s}{a+c \cos 2 s+b \sin 2 s}\right) d s}
$$

Moreover, this limit cycle is a stable hyperbolic limit cycle. 
Proof. In order to prove our results we write the polynomial differential system 1.2 in polar coordinates $(r, \theta)$, defined by $x=r \cos \theta$, and $y=r \sin \theta$, then system becomes

$$
\left\{\begin{array}{l}
r^{\prime}=(m+l \cos 2 \theta+n \sin 2 \theta) r^{3}+(a+b \sin 2 \theta+c \cos 2 \theta) r^{5} \\
\theta^{\prime}=2(a+c \cos 2 \theta+b \sin 2 \theta) r^{4}
\end{array}\right.
$$

where $\theta^{\prime}=\frac{d \theta}{d t}$ and $r^{\prime}=\frac{d r}{d t}$ According to $a+|c|+|b|<0, \theta^{\prime}$ is negative for all $t$, which means that the orbits $(r(t), \theta(t))$ of system 2.1 have the opposite orientation with respect to those $(x(t), y(t))$ of system 1.2.

Taking as new independent variable the coordinate $\theta$, this differential system writes

$$
\frac{d r}{d \theta}=\frac{1}{2} r+\frac{(m+l \cos 2 \theta+n \sin 2 \theta)}{2(a+c \cos 2 \theta+b \sin 2 \theta)} \frac{1}{r}
$$

which is a Bernoulli equation.

By introducing the standard change of variables $\rho=r^{2}$ we obtain the linear equation

$$
\frac{d \rho}{d \theta}=\rho+\frac{m+l \cos 2 \theta+n \sin 2 \theta}{a+c \cos 2 \theta+b \sin 2 \theta}
$$

The general solution of linear equation 2.3 is

$$
\rho(\theta)=e^{\theta}\left(k+\int_{0}^{\theta}\left(e^{-s} \frac{m+l \cos 2 s+n \sin 2 s}{a+c \cos 2 s+b \sin 2 s}\right) d s\right)
$$

where $k \in \mathbb{R}$

Then

$$
r(\theta)=e^{\frac{1}{2} \theta} \sqrt{k+\int_{0}^{\theta}\left(e^{-s} \frac{m+l \cos 2 s+n \sin 2 s}{a+c \cos 2 s+b \sin 2 s}\right) d s}
$$

where $k \in \mathbb{R}$

It is easy to check that the solution $r\left(\theta ; r_{0}\right)$ of the differential equation 2.2 such that $r\left(0 ; r_{0}\right)=r_{0}$ is

$$
r\left(\theta ; r_{0}\right)=e^{\frac{1}{2} \theta} \sqrt{r_{0}^{2}+\int_{0}^{\theta}\left(e^{-s} \frac{m+l \cos 2 s+n \sin 2 s}{a+c \cos 2 s+b \sin 2 s}\right) d s}
$$

where $r_{0}=r(0)$. 
A periodic solution of system 2.1 must satisfy the condition $r\left(2 \pi, r_{0}\right)=r\left(0, r_{0}\right)$, which leads to a unique value $r_{0}=r_{*}$ given by

$$
r_{*}=\sqrt{\frac{e^{2 \pi}}{e^{2 \pi}-1}} \sqrt{\int_{0}^{2 \pi}-\left(e^{-s} \frac{m+l \cos 2 s+n \sin 2 s}{a+c \cos 2 s+b \sin 2 s}\right) d s}
$$

Since $a+|c|+|b|<0, m-|l|-|n|>0$, we have $a+b \cos 2 s+c \sin 2 s<0$ and $m+l \cos 2 s+n \sin 2 s>0$ for all $s \in[0,2 \pi]$, hence $r_{*}>0$. Injecting this value of $r_{*}$ in 2.6 , we get the candidate solution

$$
\begin{aligned}
r\left(\theta ; r_{*}\right)=e^{\frac{1}{2} \theta}\left(\frac{e^{2 \pi}}{e^{2 \pi}-1} \int_{0}^{2 \pi}\right. & -\left(e^{-s} \frac{m+l \cos 2 s+n \sin 2 s}{a+c \cos 2 s+b \sin 2 s}\right) d s \\
& \left.+\int_{0}^{\theta}\left(e^{-s} \frac{m+l \cos 2 s+n \sin 2 s}{a+c \cos 2 s+b \sin 2 s}\right) d s\right)^{\frac{1}{2}}
\end{aligned}
$$

So, if $r\left(\theta ; r_{*}\right)>0$ for all $\theta \in \mathbb{R}$, we shall have $r\left(\theta ; r_{*}\right)>0$ would be periodic orbit, and consequently a limit cycle. In what follows it is proved that $r\left(\theta ; r_{*}\right)>0$ for all $\theta \in \mathbb{R}$. Indeed

$$
\begin{aligned}
r\left(\theta ; r_{*}\right)=e^{\frac{1}{2} \theta}\left(\left(\frac{1}{1-e^{2 \pi}}\right)\right. & \int_{0}^{2 \pi}\left(e^{-s} \frac{m+l \cos 2 s+n \sin 2 s}{a+c \cos 2 s+b \sin 2 s}\right) d s \\
& \left.-\int_{\theta}^{2 \pi}\left(e^{-s} \frac{m+l \cos 2 s+n \sin 2 s}{a+c \cos 2 s+b \sin 2 s}\right) d s\right)^{\frac{1}{2}}>0
\end{aligned}
$$

because $a+|c|+|b|<0, m-|l|-|n|>0$, hence $\frac{m+l \cos 2 s+n \sin 2 s}{a+c \cos 2 s+b \sin 2 s}<0$, and $\int_{0}^{2 \pi}\left(e^{-s} \frac{m+l \cos 2 s+n \sin 2 s}{a+c \cos 2 s+b \sin 2 s}\right) d s<0$ for all $s \in \mathbb{R}$

Consequently, this is a limit cycle for the differential system 1.2.

The curve $(r(\theta) \cos \theta, r(\theta) \sin (\theta))$ in the $(x, y)$ plane with

$$
r\left(\theta ; r_{*}\right)^{2}=e^{\theta}\left(r_{*}^{2}+\int_{0}^{\theta}\left(e^{-s} \frac{m+l \cos 2 s+n \sin 2 s}{a+c \cos 2 s+b \sin 2 s}\right) d s\right)
$$

is not algebraic, due to the expression $e^{\arctan \left(\frac{y}{x}\right)} r_{*}^{2}$. More precisely, in Cartesian coordinates $\left(r\left(\theta ; r_{*}\right)^{2}=x^{2}+y^{2}\right.$ and $\left.\theta=\arctan \left(\frac{y}{x}\right)\right)$, the curve defined by this limit cycle is

$$
f(x, y)=x^{2}+y^{2}-e^{\arctan \left(\frac{y}{x}\right)}\left(r_{*}^{2}+\int_{0}^{\arctan \left(\frac{y}{x}\right)}\left(e^{-s} \frac{m+l \cos 2 s+n \sin 2 s}{a+c \cos 2 s+b \sin 2 s}\right) d s\right)
$$




$$
=0 \text {. }
$$

But there is no integer $n$ for which both $\frac{\partial^{(n)} f}{\partial x^{n}}$ and $\frac{\partial^{(n)} f}{\partial y^{n}}$ vanish identically. To be convinced by this fact, one has to compute for example $\frac{\partial f}{\partial x}$, that is

$$
\begin{aligned}
\frac{\partial f}{\partial x}(x, y) & =2 x+y \frac{e^{\arctan \left(\frac{y}{x}\right)}}{x^{2}+y^{2}}\left(r_{*}^{2}+\int_{0}^{\arctan \left(\frac{y}{x}\right)}\left(e^{-s} \frac{m+l \cos 2 s+n \sin 2 s}{a+c \cos 2 s+b \sin 2 s}\right) d s\right) \\
& -\left(y \frac{-m-l\left(\cos \left(2 \arctan \left(\frac{y}{x}\right)\right)\right)-n\left(\sin \left(2 \arctan \left(\frac{y}{x}\right)\right)\right)}{\left(x^{2}+y^{2}\right)\left(a+c \cos \left(2 \arctan \left(\frac{y}{x}\right)\right)+b \sin \left(2 \arctan \left(\frac{y}{x}\right)\right)\right)}\right)
\end{aligned}
$$

Since $f(x, y)$ appears again, it will remains in any order of derivation, therefore the curve $f(x, y)=0$ is non-algebraic and the limit cycle will also be nonalgebraic.

In order to prove the hyperbolicity of the limit cycle notice that the Poincaré return map is $\Pi\left(\rho_{0}\right)=\rho\left(2 \pi, \rho_{0}\right)$, for more details see [5, Section 1.6].

An esay computation shows that

$$
\left.\frac{d r\left(2 \pi ; r_{0}\right)}{d r_{0}}\right|_{r_{0}=r_{*}}=e^{2 \pi}>1
$$

Therefore the limit cycle of the differential equation 2.2 is unstable and hyperbolic, Consequently, this is a stable and hyperbolic limit cycle for the differential system 1.2.

The following examples are given to illustrate our result.

Example 1. if we take $a=-3, b=c=m=1$, and $n=l=0$, the system 1.2 reads

$$
\left\{\begin{array}{l}
x^{\prime}=x-2(x-2 y)\left(x^{2}-x y+2 y^{2}\right) \\
y^{\prime}=y-2(2 x+y)\left(x^{2}-x y+2 y^{2}\right)
\end{array}\right.
$$

is a cubic system has a non-algebraic limit cycle explicitly given

$$
r\left(\theta ; r_{*}\right)=e^{\frac{1}{2} \theta} \sqrt{r_{*}^{2}+\int_{0}^{\theta}\left(\frac{e^{-s}}{-3+\cos 2 s+\sin 2 s}\right) d s}
$$

where $\theta \in \mathbb{R}$, and $r_{*} \simeq 0.68591$ 
Example 2. if we take $a=-3, b=1, c=0, n=3, m=8$, and $l=2$, the system 1.2 reads

$$
\left\{\begin{array}{l}
x^{\prime}=x\left(10 x^{2}+6 x y+6 y^{2}\right)+(x-2 y)\left(x^{2}+y^{2}\right)\left(-3 x^{2}+2 x y-3 y^{2}\right) \\
y^{\prime}=y\left(10 x^{2}+6 x y+6 y^{2}\right)+(2 x+y)\left(x^{2}+y^{2}\right)\left(-3 x^{2}+2 x y-3 y^{2}\right)
\end{array}\right.
$$

is a cubic system has a non-algebraic limit cycle explicitly given

$$
r\left(\theta ; r_{*}\right)=e^{\frac{1}{2} \theta} \sqrt{r_{*}^{2}+\int_{0}^{\theta}\left(e^{-s} \frac{8+2 \cos 2 s+3 \sin 2 s}{-3+\sin 2 s}\right) d s}
$$

where $\theta \in \mathbb{R}$, and $r_{*} \simeq 1.9923$

Example 3. if we take $a=-3, b=c=n=l=1$, and $m=2$, then system 1.2 reads

$$
\left\{\begin{array}{l}
x^{\prime}=x\left(3 x^{2}+2 x y+y^{2}\right)-2(x-2 y)\left(x^{2}+y^{2}\right)\left(x^{2}-x y++2 y^{2}\right) \\
y^{\prime}=y\left(3 x^{2}+2 x y+y^{2}\right)-2(2 x+y)\left(x^{2}+y^{2}\right)\left(x^{2}-x y++2 y^{2}\right)
\end{array}\right.
$$

is a quintic system has a non-algebraic limit cycle explicitly given

$$
r\left(\theta ; r_{*}\right)=e^{\frac{1}{2} \theta} \sqrt{r_{*}^{2}+\int_{0}^{\theta}\left(e^{-s} \frac{2+\cos 2 s+\sin 2 s}{-3+\cos 2 s+\sin 2 s}\right) d s}
$$

where $\theta \in \mathbb{R}$, and $r_{*} \simeq 1.1629$

Example 4. if we take $a=-4, b=c=1, n=-1, m=5$, and $l=2$, then system 1.2 reads

$$
\left\{\begin{array}{l}
x^{\prime}=x\left(7 x^{2}-2 x y+3 y^{2}\right)+(x-2 y)\left(x^{2}+y^{2}\right)\left(-3 x^{2}+2 x y-5 y^{2}\right) \\
y^{\prime}=y\left(7 x^{2}-2 x y+3 y^{2}\right)+(2 x+y)\left(x^{2}+y^{2}\right)\left(-3 x^{2}+2 x y-5 y^{2}\right)
\end{array}\right.
$$

is a quintic system has a non-algebraic limit cycle explicitly given

$$
r\left(\theta ; r_{*}\right)=e^{\frac{1}{2} \theta} \sqrt{r_{*}^{2}+\int_{0}^{\theta}\left(e^{\left.-s \frac{5+2 \cos 2 s-\sin 2 s}{-4+\cos 2 s+\sin 2 s}\right)} d s\right.}
$$

where $\theta \in \mathbb{R}$, and $r_{*} \simeq 1.2742$ 


\section{References}

[1] Al-Dosary, Khalil I. T. Non-algebraic limit cycles for parametrized planar polynomial systems, Int. J. Math 18, No. 2, 179-189 (2007).

[2] A. Bendjeddou and R. Cheurfa, On the exact limit cycle for some class of planar differential systems, Nonlinear differ. equ. appl. 14 (2007), 491-498.

[3] A. Bendjeddou and R. Cheurfa, Cubic and quartic planar differential systems with exact algebraic limit cycles, Elect. J. of Diff. Equ., no15 (2011), $1-12$.

[4] R. Benterki and J. Llibre, Polynomial differential systems with explicit non-algebraic limit cycles, Elect. J. of Diff. Equ., no78 (2012), 1-6.

[5] F. Dumortier, J. Llibre and J. Artés, Qualitative Theory of Planar Differential Systems, (Universitex) Berlin, Springer (2006).

[6] A. Gasull, H. Giacomini and J. Torregrosa, Explicit non-algebraic limit cycles for polynomial systems, J. Comput. Appl. Math. 200 (2007) 448457.

[7] J. Giné and M. Grau, Coexistence of algebraic and non-algebraic limit cycles, explicitly given, using Riccati equations, Nonlinearity 19 (2006) 19391950 .

[8] H. Giacominiy, J. Llibre and M. Viano, On the nonexistence existence and uniquencess of limit cycles, Nonlinearity 9 (1996) 501-516. Printed in the $\mathrm{UK}$

[9] J. Llibre and Y. Zhao, Algebraic Limit Cycles in Polynomial Systems of Differential Equations, J. Phys. A: Math. Theor. 40 (2007), 14207-14222.

[10] K. Odani, The limit cycle of the van der Pol equation is not algebraic, J. of Diff. Equ. 115 (1995), 146-152. 
